\title{
Combined Transceiver Optimization for Uplink Multiuser MIMO with Limited CSI
}

\author{
Chia-Chang Hu and Chung-Lun Yang \\ Department of Communications Engineering, National Chung Cheng University, 168 University Road, \\ Min-Hsiung, Chiayi 621, Taiwan \\ Correspondence should be addressed to Chia-Chang Hu, ieecch@ccu.edu.tw
}

Received 23 December 2010; Accepted 12 January 2011

Academic Editors: S. K. Bhatia and M. A. Nappi

Copyright (๑) 2011 C.-C. Hu and C.-L. Yang. This is an open access article distributed under the Creative Commons Attribution License, which permits unrestricted use, distribution, and reproduction in any medium, provided the original work is properly cited.

Joint precoder and decoder optimization is considered for uplink multiuser multiple-input multiple-output (MU-MIMO) systems with limited channel state information (CSI) at both the transmitters and receivers. Instead of counting on complex iterativebased algorithms, an efficient and noniterative QR-based linear transceiver pair design is employed. In addition, an equal power distribution (EPD) scheme is applied to adjust transmit power allocation of each mobile station (MS) between its symbols under the total transmit-power constraint. Simulations are conducted to provide a comparative evaluation of the proposed QREPD algorithm with other transceiver designs on the sum mean-squared error (SMSE) and the averaged bit-error-rate (BER) performance.

\section{Introduction}

Owing to the emerging demand on multimedia applications, next-generation wireless communication systems are expected to improve substantially both reliability and spectral efficiency. A key development in this regard is the use of multiple antenna arrays at both the base station (BS) and mobile station (MS) [1]. Due to its effectiveness in coping with multiple-access interference (MAI) and interstream interference (ISI), a linear precoder-decoder joint architecture is utilized based on the minimum sum meansquared error (MSMSE) to improve multiuser multipleinput multiple-output (MU-MIMO) spatial multiplexing systems [2-4]. To further enhance system performance of MU-MIMO systems, one important strategy is to exploit feedback information at the transmitter. In particular, when channel state information (CSI) is fully known at the transmitter, spatial multiplexing is able to benefit significantly from the use of precoding at the transmitter [2]. In most previous research, the perfect CSI is available at both ends [5, 6]. However, the knowledge of CSI is in general incomplete in reality due to various kinds of channel imperfections, such as channel estimation error, feedback delay, and finite-rate channel equalization. In addition, the development of the transceiver depends primarily on the complex iterativebased algorithms, in which they either suffer from a slow convergence rate at high signal-to-noise ratio (SNR) or may even not converge to global optimum [7]. Therefore, instead of relying on complicated recursive-based algorithms, an efficient noniterative QR-based transceiver design is proposed for uplink MU-MIMO systems under limited CSI at both sides. Moreover, an equal power distribution (EPD) technique is applied to allocate uniformly the transmission power of each mobile user between its symbols under a fixed total transmit power. Thus, the superiority of the proposed QR-EPD scheme on the MSMSE and the averaged BER performance for uplink MU-MIMO systems can be achieved.

The rest of the paper is organized as follows. Section 2 describes the uplink multiuser MIMO system and channel model. In Section 3, the transceiver-pair design of the uplink multiuser MIMO systems is formulated as the constrained sum-MSE optimization problem. An efficient noniterative QR-based linear transceiver architecture is detailed for uplink multiuser MIMO systems with limited CSI in Section 4. A simple EPD scheme is applied to allocate uniformly the transmission power of each symbol of the 
mobile user under the sum-power constraint. Numerical results and conclusions are presented in Sections 5 and 6, respectively.

Notation. Symbols for matrices (vectors) are denoted by boldface upper (lower) case letters. The superscripts $(\cdot)^{\star}$ and $(\cdot)^{\mathrm{H}}$ stand for complex conjugation and Hermitian transposition, respectively. $E\{\cdot\}$ denotes the expected-value operator. $\|\cdot\|$ indicates the matrix/vector Frobenius norm. $\mathbf{I}_{M}$ is an $M \times M$ identity matrix. $\operatorname{tr}(\cdot)$ and $[\cdot]_{i, j}$ denote, respectively, the trace and the $(i, j)$ th entry of a matrix. Finally, $(x)_{+}$stands for $\max (x, 0)$.

\section{Uplink MIMO System and Channel Model}

Consider an uplink multiuser MIMO wireless system with an $M$-antenna BS and $K$ mobile users each with $N$ antennas, as shown in Figure 1. The uplink MIMO channel of the $i$ th user is denoted by $\mathbf{H}_{i} \in C^{M \times N}, i=1,2, \ldots, K$. Two important constraints need to be satisfied in the scenario to guarantee resolvability. First of all, there must have at least as many receiver antennas at BS as the total number of transmit antennas of all mobile users, that is, $K N \leq M$. As well, each user must have as many transmit antennas as the transmitted data streams. Suppose that user $i$ has data streams, denoted by $\mathbf{x}_{i} \in C^{N \times 1}, i=1,2, \ldots, K$, which are assumed to be zero-mean white random vectors each with unit-energy $E\left\{\mathbf{x}_{i} \mathbf{x}_{i}^{\mathrm{H}}\right\}=\mathbf{I}_{N}$, for all $i$ and mutually statistically independent among users. A linear precoder is employed for each user, which is denoted by $\mathbf{F}_{i} \in C^{N \times N}, i=1,2, \ldots, K$. The received signal vector $\mathbf{r} \in C^{M \times 1}$ at the $\mathrm{BS}$ is given by

$$
\mathbf{r}=\sum_{i=1}^{K} \mathbf{H}_{i} \mathbf{F}_{i} \mathbf{x}_{i}+\mathbf{n},
$$

where the noise vector $\mathbf{n}$ is modeled as zero-mean white complex Gaussian variables, that is, $C N\left(0, \sigma_{\mathrm{n}}^{2} \mathbf{I}_{M}\right)$. The data and the noise are assumed to be statistically independent. The received signal vector $\mathbf{r}$ is processed by all linear decoders $\mathbf{G}_{j} \in C^{N \times M}$, for $j=1,2, \ldots, K$. An estimate of the data vector for user $j$ can be expressed as

$$
\mathbf{y}_{j}=\mathbf{G}_{j} \mathbf{r}=\mathbf{G}_{j}\left(\sum_{i=1}^{K} \mathbf{H}_{i} \mathbf{F}_{i} \mathbf{x}_{i}+\mathbf{n}\right) .
$$

The MIMO channels among users are assumed to be independent and flat fading. The uplink CSI model at the BS can be expressed as

$$
\mathbf{H}_{i}=\hat{\mathbf{H}}_{i}+\Delta \mathbf{H}_{i}, \quad i=1,2, \ldots, K,
$$

where $\hat{\mathbf{H}}_{i}$ is the estimated channel matrix and $\Delta \mathbf{H}_{i}$ is the CSI-error matrix. The entries of $\mathbf{H}_{i}, \hat{\mathbf{H}}_{i}$, and $\Delta \mathbf{H}_{i}$ are identically independent distribution (i.i.d.) complex Gaussian variables each with $C N(0,1), C N\left(0,1-\sigma_{\mathrm{E}}^{2}\right)$, and $C N\left(0, \sigma_{\mathrm{E}}^{2}\right)$, respectively. Here, $\sigma_{\mathrm{E}}^{2}$ indicates the channel estimation-error variance for each mobile user. Furthermore, matrices $\hat{\mathbf{H}}_{i}$ and $\Delta \mathbf{H}_{i}$ are assumed to be uncorrelated.

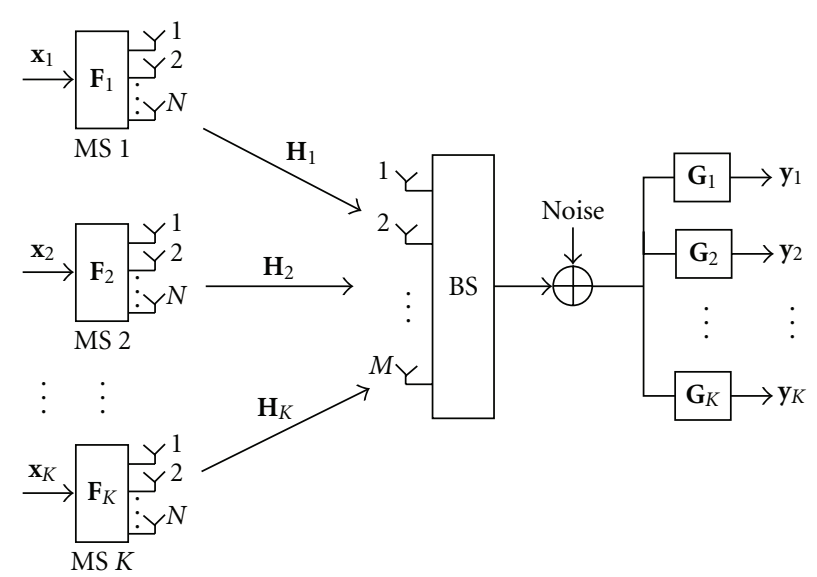

FIGURE 1: Block diagram of an uplink multiuser MIMO wireless system with an $M$-antenna BS and $K$ mobile users each with $N$ antennas.

\section{The Uplink MIMO Problem Formulation}

With the CSI model, the received signal vector at the BS is written as

$$
\mathbf{r}=\sum_{i=1}^{K}\left(\hat{\mathbf{H}}_{i}+\Delta \mathbf{H}_{i}\right) \mathbf{F}_{i} \mathbf{x}_{i}+\mathbf{n},
$$

and the estimate of the data vector for user $j$ is expressed as

$$
\mathbf{y}_{j}=\mathbf{G}_{j}\left[\sum_{i=1}^{K}\left(\hat{\mathbf{H}}_{i}+\Delta \mathbf{H}_{i}\right) \mathbf{F}_{i} \mathbf{x}_{i}+\mathbf{n}\right] .
$$

The MSE matrix for user $j$ is defined by

$$
\begin{aligned}
\operatorname{MSE}_{j}= & E\left\{\left(\mathbf{y}_{j}-\mathbf{x}_{j}\right)\left(\mathbf{y}_{j}-\mathbf{x}_{j}\right)^{\mathrm{H}}\right\} \\
= & \mathbf{G}_{j}\left(\sum_{i=1}^{K} \hat{\mathbf{H}}_{i} \mathbf{F}_{i} \mathbf{F}_{i}^{\mathrm{H}} \hat{\mathbf{H}}_{i}^{\mathrm{H}}+\sigma_{\mathrm{n}}^{2} \mathbf{I}_{M}\right) \mathbf{G}_{j}^{\mathrm{H}} \\
& +\mathbf{G}_{j}\left[\sum_{i=1}^{K} E\left\{\Delta \mathbf{H}_{i} \mathbf{F}_{i} \mathbf{F}_{i}^{\mathrm{H}} \Delta \mathbf{H}_{i}^{\mathrm{H}}\right\}\right] \mathbf{G}_{j}^{\mathrm{H}} \\
& -\mathbf{G}_{j} \hat{\mathbf{H}}_{j} \mathbf{F}_{j}-\mathbf{F}_{j}^{\mathrm{H}} \hat{\mathbf{H}}_{j}^{\mathrm{H}} \mathbf{G}_{j}^{\mathrm{H}}+\mathbf{I}_{N} .
\end{aligned}
$$

Then it can be shown that

$$
\begin{aligned}
\operatorname{MSE}_{j}= & \mathbf{G}_{j}\left(\sum_{i=1}^{K} \hat{\mathbf{H}}_{i} \mathbf{F}_{i} \mathbf{F}_{i}^{\mathrm{H}} \hat{\mathbf{H}}_{i}^{\mathrm{H}}+\sigma_{\mathrm{n}}^{2} \mathbf{I}_{M}\right) \mathbf{G}_{j}^{\mathrm{H}} \\
& +\mathbf{G}_{j}\left[\sum_{i=1}^{K} \operatorname{tr}\left(\mathbf{F}_{i} \mathbf{F}_{i}^{\mathrm{H}}\right) \cdot \sigma_{\mathrm{E}}^{2} \mathbf{I}_{M}\right] \mathbf{G}_{j}^{\mathrm{H}} \\
& -\mathbf{G}_{j} \hat{\mathbf{H}}_{j} \mathbf{F}_{j}-\mathbf{F}_{j}^{\mathrm{H}} \hat{\mathbf{H}}_{j}^{\mathrm{H}} \mathbf{G}_{j}^{\mathrm{H}}+\mathbf{I}_{N},
\end{aligned}
$$

where the equality of

$$
E\left\{\Delta \mathbf{H}_{i} \mathbf{F}_{i} \mathbf{F}_{i}^{\mathrm{H}} \Delta \mathbf{H}_{i}^{\mathrm{H}}\right\}=\operatorname{tr}\left(\mathbf{F}_{i} \mathbf{F}_{i}^{\mathrm{H}}\right) \cdot \sigma_{\mathrm{E}}^{2} \mathbf{I}_{M}
$$


is used in (7). The sum-MSE of all users is given by

$$
\mathrm{MSE}=\sum_{j=1}^{K} \operatorname{tr}\left(\mathrm{MSE}_{j}\right)
$$

The uplink MU-MIMO problem is to minimize the sumMSE subject to (s.t.) the sum-power constraint, which is given as follows:

$$
\begin{array}{rl}
\min _{\left\{\left(\mathbf{F}_{j}, \mathbf{G}_{j}\right)\right\}_{j=1}^{K}} & \mathrm{MSE}=\sum_{j=1}^{K} \operatorname{tr}\left(\mathrm{MSE}_{j}\right), \\
\text { s.t. } & \sum_{j=1}^{K} \operatorname{tr}\left(\mathbf{F}_{j} \mathbf{F}_{j}^{\mathrm{H}}\right)=P,
\end{array}
$$

where $P$ is the total transmit power constraint over all users, that is, the sum-power constraint. To find the joint optimum set of linear precoders and decoders, one can devise iterativebased algorithms that decrease monotonically the total MSE. Similar algorithms have been proposed in [2-4] with perfect CSI. In summary, such a set of transceivers are derived by using the Lagrange multipliers associated with the transmit power constraint in (11), which is given as follows:

$$
\min _{\left\{\left(F_{j}, G_{j}\right)\right\}_{j=1}^{K}, \mu} \mathrm{MSE}+\mu\left[\sum_{j=1}^{K} \operatorname{tr}\left(\mathbf{F}_{j} \mathbf{F}_{j}^{\mathrm{H}}\right)-P\right] .
$$

The scalar $\mu$ in (12) is the Lagrangian multiplier. Similar to [7], a noniterative set of linear precoders and decoders are utilized by relaxing a nonconvex optimization problem in (10) into a simpler convex optimization problem. Thus, two traditional issues of the complexity cost and the convergence speed in most iterative-based algorithms can be avoided.

\section{MU-MIMO Transceiver Design}

Given a set of precoders $\mathbf{F}_{i}, i=1,2, \ldots, K$, the optimal linear decoder $\mathbf{G}_{j}$ can be obtained by taking derivative of (9) with respect to $\mathbf{G}_{j}^{\star}, j=1,2, \ldots, K$, and then equating it to zero. Therefore, the optimal linear decoder $\mathbf{G}_{j}$ is obtained by [3]

$$
\mathbf{G}_{j}=\mathbf{F}_{j}^{\mathrm{H}} \hat{\mathbf{H}}_{j}^{\mathrm{H}}\left(\rho \mathbf{I}_{M}+\sum_{i=1}^{K} \hat{\mathbf{H}}_{i} \mathbf{F}_{i} \mathbf{F}_{i}^{\mathrm{H}} \hat{\mathbf{H}}_{i}^{\mathrm{H}}\right)^{-1},
$$

where

$$
\rho=\left[\sigma_{\mathrm{n}}^{2}+\sum_{i=1}^{K} \sigma_{\mathrm{E}}^{2} \cdot \operatorname{tr}\left(\mathbf{F}_{i} \mathbf{F}_{i}^{\mathrm{H}}\right)\right]
$$

is used in (13). For convenience of notation, the matrix $\mathbf{T}$ is defined as follows:

$$
\mathbf{T}=\rho \mathbf{I}_{M}+\sum_{i=1}^{K} \hat{\mathbf{H}}_{i} \mathbf{F}_{i} \mathbf{F}_{i}^{\mathrm{H}} \hat{\mathbf{H}}_{i}^{\mathrm{H}} .
$$

Then, substituting (13) into (9) derives a simplified SMSE expression given by

$$
\begin{aligned}
\text { MSE } & =\rho \cdot \operatorname{tr}\left(\mathbf{T}^{-1}\right)+\sum_{j=1}^{K} \operatorname{tr}\left(\mathbf{I}_{N}\right)-\operatorname{tr}\left(\mathbf{I}_{M}\right) \\
& =\operatorname{tr}\left\{\left(\mathbf{I}_{M}+\frac{1}{\rho} \sum_{i=1}^{K} \hat{\mathbf{H}}_{i} \mathbf{F}_{i} \mathbf{F}_{i}^{\mathrm{H}} \hat{\mathbf{H}}_{i}^{\mathrm{H}}\right)^{-1}\right\}+K N-M .
\end{aligned}
$$

Therefore, the uplink MU-MIMO problem can be equivalently formulated as

$$
\begin{aligned}
\min _{\left\{\mathbf{F}_{i}\right\}_{i=1}^{K}} \operatorname{MSE}= & \operatorname{tr}\left\{\left(\mathbf{I}_{M}+\frac{1}{\rho} \tilde{\mathbf{H}} \tilde{\mathbf{F}} \tilde{\mathbf{H}}^{\mathrm{H}}\right)^{-1}\right\} \\
& +K N-M \\
\text { s.t. } \quad \operatorname{tr}(\tilde{\mathbf{F}})= & P,
\end{aligned}
$$

where the matrix $\tilde{\mathbf{H}} \tilde{\mathbf{F}} \tilde{\mathbf{H}}^{\mathrm{H}}$ in (18) is employed to represent the matrix $\sum_{i=1}^{K} \hat{\mathbf{H}}_{i} \mathbf{F}_{i} \mathbf{F}_{i}^{\mathrm{H}} \hat{\mathbf{H}}_{i}^{\mathrm{H}}$ in (17) and the relationships between both matrices are given by

$$
\begin{gathered}
\widetilde{\mathbf{H}}=\left[\hat{\mathbf{H}}_{1} \hat{\mathbf{H}}_{2} \cdots \hat{\mathbf{H}}_{K}\right] \\
\widetilde{\mathbf{F}}=\left[\begin{array}{cccc}
\mathbf{F}_{1} \mathbf{F}_{1}^{\mathrm{H}} & \mathbf{0} & \cdots & \mathbf{0} \\
\mathbf{0} & \mathbf{F}_{2} \mathbf{F}_{2}^{\mathrm{H}} & \cdots & \mathbf{0} \\
\vdots & \vdots & \ddots & \vdots \\
\mathbf{0} & \mathbf{0} & \cdots & \mathbf{F}_{K} \mathbf{F}_{K}^{\mathrm{H}}
\end{array}\right] .
\end{gathered}
$$

Note that no cooperation is assumed among mobile users in the MU-MIMO system. Therefore, the matrix $\widetilde{\mathbf{F}}$ possesses a block diagonal structure as defined in (21). Unfortunately, no simple closed-form solution can be achieved for such a block diagonal constraint. In order to obtain the optimal solution of $\widetilde{\mathbf{F}}$, the block-diagonal constraint on $\widetilde{\mathbf{F}}$ needs to be relaxed for this moment. In what follows, the matrix of $\widetilde{\mathbf{F}}^{\text {opt }}$ is used to indicate the optimal solution of $\widetilde{\mathbf{F}}$. Instead of the singular value decomposition (SVD) used to decompose the matrix $\tilde{\mathbf{H}}$ in [7], the computationally efficient $Q R$ factorization is employed to perform the decomposition of the matrix $\tilde{\mathbf{H}}^{\mathrm{H}} \tilde{\mathbf{H}}$ as follows:

$$
\mathbf{Q}_{\widetilde{\mathbf{H}}}^{\mathrm{H}} \tilde{\mathbf{H}}^{\mathrm{H}} \tilde{\mathbf{H}}=\mathbf{R}_{\widetilde{\mathbf{H}}},
$$

where the $K N \times K N$ matrix $\mathbf{Q}_{\widetilde{\mathbf{H}}}^{\mathrm{H}}$ is a unitary matrix with $\mathbf{Q}_{\widetilde{\mathbf{H}}}^{-1}=\mathbf{Q}_{\widetilde{\mathbf{H}}}^{\mathrm{H}}$ that takes the matrix $\widetilde{\mathbf{H}}^{\mathrm{H}} \tilde{\mathbf{H}}$ to an upper triangular form of $\mathbf{R}_{\widetilde{\mathbf{H}}}$ with dimension $K N \times K N$. As a consequence, the QR-based scheme in $[8,9]$ can be utilized to find approximations of eigenvalues of $\widetilde{\mathbf{H}}^{\mathrm{H}} \widetilde{\mathbf{H}}$, that is,

$$
\Lambda_{\widetilde{\mathbf{H}}}=\mathbf{Q}_{\widetilde{\mathbf{H}}}^{\mathrm{H}} \tilde{\mathbf{H}}^{\mathrm{H}} \tilde{\mathbf{H}} \mathbf{Q}_{\tilde{\mathbf{H}}} \text {. }
$$

Subsequently, the diagonal elements of $\boldsymbol{\Lambda}_{\widetilde{\mathbf{H}}}$ is rearranged in a decreasing order. Finally, the matrix $\widetilde{\mathbf{F}}^{\text {opt }}$ can be attained by $[5,7,10]$

$$
\widetilde{\mathbf{F}}^{\text {opt }}=\mathbf{Q}_{\widetilde{\mathbf{H}}} \boldsymbol{\Sigma}_{f} \mathbf{Q}_{\widetilde{\mathbf{H}}}^{\mathrm{H}} \text {, }
$$


where the diagonal elements $\left[\Sigma_{f}\right]_{i, i}, i=1,2, \ldots, K N$, of the matrix $\Sigma_{f}$ are determined by [7]

$$
\left[\Sigma_{f}\right]_{i, i}=\left(\sqrt{\frac{\rho}{\mu\left[\boldsymbol{\Lambda}_{\tilde{\mathbf{H}}}\right]_{i, i}}}-\frac{\rho}{\left[\boldsymbol{\Lambda}_{\tilde{\mathbf{H}}}\right]_{i, i}}\right)_{+} .
$$

As the matrix $\widetilde{\mathbf{F}}^{\text {opt }}$ is derived, the $i$ th block diagonal elements of $\tilde{\mathbf{F}}^{\text {opt }}$, for $i=1,2, \ldots, K$, can be acquired by using

$$
\mathbf{F}_{i} \mathbf{F}_{i}^{\mathrm{H}}=\left[\widetilde{\mathbf{F}}^{\mathrm{opt}}\right]_{(i-1) N+1: i N,(i-1) N+1: i N},
$$

while the condition of the total sum-power constraint has to be preserved simultaneously. In general, the precoders $\mathbf{F}_{i}$, $i=1,2, \ldots, K$, are obtained by performing Cholesky factorizations of the matrix $\mathbf{F}_{i} \mathbf{F}_{i}^{\mathrm{H}}, i=1,2, \ldots, K$. Here, a simple and novel scheme is proposed to perform factorization of $\mathbf{F}_{i} \mathbf{F}_{i}^{\mathrm{H}}$ to obtain suboptimal precoders, denoted by $\widehat{\mathbf{F}}_{i}, i=1,2, \ldots, K$. Note that every multisymbol transmission of each user can be viewed as multiple single symbol transmissions of each user and each symbol has its corresponding column in the linear precoder matrix. Thus, our aim is now to directly impose power constraints on the columns of the precoder matrix. The question is how to obtain the precoder matrix that satisfies the predetermined total power constraint of each mobile user, that is, $\operatorname{tr}\left(\widehat{\mathbf{F}}_{i} \hat{\mathbf{F}}_{i}^{\mathrm{H}}\right)=\operatorname{tr}\left(\mathbf{F}_{i} \mathbf{F}_{i}^{\mathrm{H}}\right)$, for $i=$ $1,2, \ldots, K$. Fortunately, there exists a method that guarantees the existence of at least one precoder matrix satisfying the given power distribution constraint. This scheme is called the EPD, which is presented as follows: for a given matrix $\mathbf{F}_{i} \mathbf{F}_{i}^{\mathrm{H}}$ in (26), there exists a transmitter matrix $\widehat{\mathbf{F}}_{i}=\left[\widehat{\mathbf{f}}_{1}, \widehat{\mathbf{f}}_{2}, \ldots, \widehat{\mathbf{f}}_{N}\right]$, such that the conditions of $\left\|\hat{\mathbf{f}}_{m}\right\|^{2}=\left\|\hat{\mathbf{f}}_{n}\right\|^{2}$, for $m, n=$ $1,2, \ldots, N$, can hold. In other words, each column vector $\hat{\mathbf{f}}_{m}$ of the matrix $\widehat{\mathbf{F}}_{i}$ has equal power. Denote $\gamma$ as the rank of the matrix $\mathbf{F}_{i} \mathbf{F}_{i}^{\mathrm{H}}$. Here, $\lambda_{k}$ and $\mathbf{u}_{k}$ are employed to indicate the $k$ th eigenvalue and the associated eigenvector of the matrix $\mathbf{F}_{i} \mathbf{F}_{i}^{\mathrm{H}}$. Thus, the $m$ th column vector of $\widehat{\mathbf{F}}_{i}$ is obtained and given by

$$
\widehat{\mathbf{f}}_{m}=\sum_{k=1}^{\gamma} \sqrt{\frac{\lambda_{k}}{N}} \cdot e^{j(2 \pi m k / N)} \cdot \mathbf{u}_{k} .
$$

Thus, the transmit power of each user is distributed equally between its symbols. The procedures of the proposed QREPD algorithm are summarized in Table 1.

\section{Numerical Results}

In this section, computer simulations are conducted to demonstrate the performance of the proposed QR-EPD transceiver design as developed in Section 4. In the simulations, the notation of $\left[K, M, N, \sigma_{\mathrm{E}}^{2}\right]$ is employed to denote a $K$-user uplink MU-MIMO system equipped with $M$ antennas at the BS and $N$ antennas at the MS. Moreover, $\sigma_{\mathrm{E}}^{2}$ is used to stand for channel estimation-error variance for each user. In addition, the 4-QAM modulation method is adopted in each user's data streams. The total transmit power equal to the number of mobile users, that is, $P=K$, is assumed. To emphasize the importance of the use of the EPD scheme,
TABLE 1: The QR-EPD noniterative algorithm.

(1) First, the QR factorization is employed to perform the decomposition of the matrix $\tilde{\mathbf{H}}^{\mathrm{H}} \tilde{\mathbf{H}}$ as follows: $\widetilde{\mathbf{H}}^{\mathrm{H}} \tilde{\mathbf{H}}=\mathbf{Q}_{\tilde{\mathbf{H}}} \mathbf{R}_{\tilde{\mathbf{H}}}$.

(2) A simple QR-based scheme [8] is utilized to find approximations of eigenvalues of $\widetilde{\mathbf{H}}^{\mathrm{H}} \widetilde{\mathbf{H}}$, that is,

$\boldsymbol{\Lambda}_{\widetilde{\mathbf{H}}}=\mathbf{Q}_{\widetilde{\mathrm{H}}}^{\mathrm{H}} \widetilde{\mathbf{H}}^{\mathrm{H}} \widetilde{\mathbf{H}} \mathbf{Q}_{\widetilde{\mathbf{H}}}$. The diagonal elements of $\boldsymbol{\Lambda}_{\widetilde{\mathbf{H}}}$ are rearranged in a decreasing order.

(3) The matrix $\widetilde{\mathbf{F}}^{\text {opt }}$ is attained by $\widetilde{\mathbf{F}}^{\text {opt }}=\mathbf{Q}_{\widetilde{\mathbf{H}}} \boldsymbol{\Sigma}_{f} \mathbf{Q}_{\widetilde{\mathbf{H}}}^{\mathrm{H}}$, where

$\left[\boldsymbol{\Sigma}_{f}\right]_{i, i}=\left(\sqrt{\frac{\rho}{\mu\left[\boldsymbol{\Lambda}_{\widetilde{\mathbf{H}}}\right]_{i, i}}}-\frac{\rho}{\left[\boldsymbol{\Lambda}_{\widetilde{\mathbf{H}}}\right]_{i, i}}\right)$.

(4) As the matrix $\widetilde{\mathbf{F}}^{\text {opt }}$ is derived, the $i$ th block diagonal elements of $\widetilde{\mathbf{F}}^{\text {opt }}$, for $i=1,2, \ldots, K$, need to be acquired, that is,

$\mathbf{F}_{i} \mathbf{F}_{i}^{\mathrm{H}}=\left[\widetilde{\mathbf{F}}^{\text {opt }}\right]_{(i-1) N+1: i N,(i-1) N+1: i N}$.

(5) Finally, the EPD scheme is utilized to perform factorization of $\mathbf{F}_{i} \mathbf{F}_{i}^{\mathrm{H}}$ to obtain suboptimal precoders $\hat{\mathbf{F}}_{i}, i=1,2, \ldots, K$,

$\hat{\mathbf{F}}_{i}=\left[\widehat{\mathbf{f}}_{1}, \hat{\mathbf{f}}_{2}, \ldots, \hat{\mathbf{f}}_{N}\right]$, where the column vector of $\widehat{\mathbf{F}}_{i}$ is given by

$\hat{\mathbf{f}}_{m}=\sum_{k=1}^{\gamma} \sqrt{\frac{\lambda_{k}}{N}} \cdot e^{j(2 \pi m k / N)} \cdot \mathbf{u}_{k}$.

the SMSE and averaged BER performance of the QR-EPD and SVD-EPD schemes are evaluated and compared with the transceiver design proposed in [7], denoted as SVD nonEPD. In both the SVD-EPD and SVD non-EPD schemes, the SVD is applied to decompose the matrix $\tilde{\mathbf{H}}$ into the matrixproduct form as follows:

$$
\widetilde{\mathbf{H}}=\mathbf{U}_{\widetilde{\mathbf{H}}} \Sigma_{\widetilde{\mathbf{H}}} \mathbf{V}_{\widetilde{\mathbf{H}}}^{\mathrm{H}},
$$

where both matrices $\mathbf{U}_{\widetilde{\mathbf{H}}}$ and $\mathbf{V}_{\widetilde{\mathbf{H}}}$ in (28) denote the $M \times M$ and $K N \times K N$ unitary matrices and $\Sigma_{\widetilde{\mathbf{H}}}$ is the diagonal matrix with singular values arranged in the decreasing order. The matrix $\widetilde{\mathbf{F}}^{\text {opt }}$ has been derived in $[5,7,10]$, which is given by

$$
\widetilde{\mathbf{F}}^{\text {opt }}=\mathbf{V}_{\widetilde{\mathbf{H}}} \Sigma_{f} \mathbf{V}_{\widetilde{\mathbf{H}}^{\mathrm{H}}}^{\mathrm{H}}
$$

where the diagonal elements $\left[\boldsymbol{\Sigma}_{f}\right]_{i, i}, i=1,2, \ldots, K N$, of the matrix $\Sigma_{f}$ are determined by [7]

$$
\left[\Sigma_{f}\right]_{i, i}=\left(\sqrt{\frac{\rho}{\mu\left[\Sigma_{\tilde{\mathbf{H}}}\right]_{i, i}^{2}}}-\frac{\rho}{\left[\Sigma_{\tilde{\mathbf{H}}}\right]_{i, i}^{2}}\right)_{+} .
$$

Also, to check out our derivations of the closed-form solution, the QR-EPD scheme is compared with an iterative transceiver structure based on the Karush-Kuhn-Tucker (KKT) optimality conditions [11].

In Figure 2, the SMSE and the averaged BER comparisons between the QR-EPD, the SVD-EPD, the SVD non-EPD, and the KKT schemes are evaluated in terms of $\mathrm{SNR}(\mathrm{dB})$ with parameters $\left[K, M, N, \sigma_{\mathrm{E}}^{2}=0\right]$, that is, the perfectly-known CSI is available. In both figures, it is observed the fact that the SMSE and the average BER performance of the proposed QR-EPD are close to the SVD-EPD and better than those of the SVD non-EPD and the KKT. Another important fact to be emphasized is that when the total number of transmit antennas of all mobile users is less than the number of 


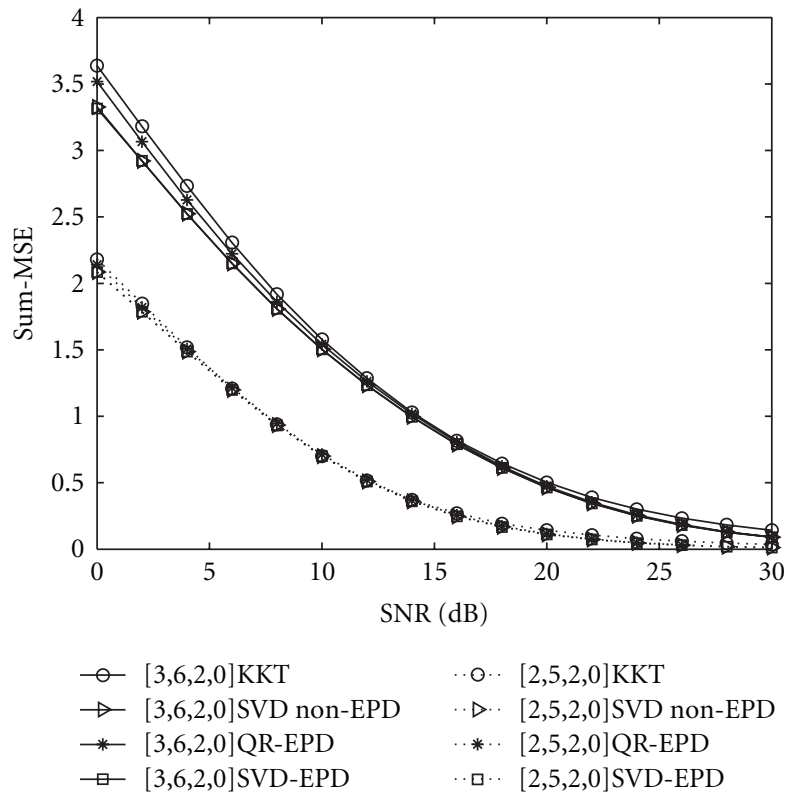

(a)

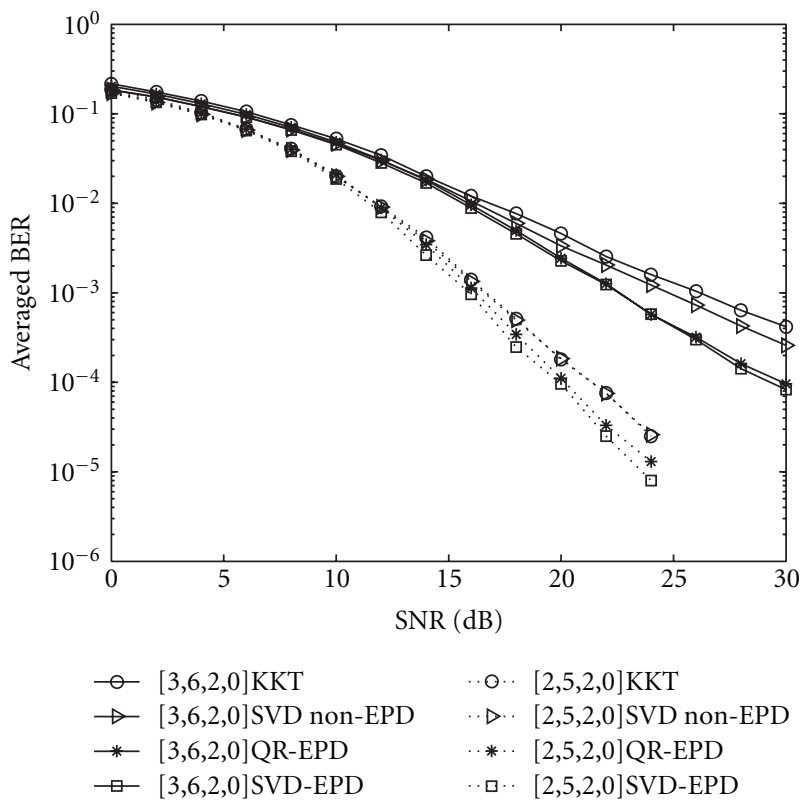

(b)

FIgURE 2: (a) The SMSE and (b) the averaged BER comparisons between the proposed QR-EPD, the SVD-EPD, the SVD non-EPD, and the KKT algorithms for $\sigma_{\mathrm{E}}^{2}=0$.

receiver antennas at $\mathrm{BS}$, that is, $K N<M$, the SMSE and the average BER performance of these four algorithms are much better than those of the same schemes with $K N=M$. This is reasonable because the utilization of $K N<M$ is able to offer a larger spatial diversity gain.

Results of Figures 3 and 4 show that the SMSE and the averaged BER performance of the proposed QR-EPD, the SVD-EPD, the SVD non-EPD, and the KKT schemes for the MIMO communication systems with the use of parameters

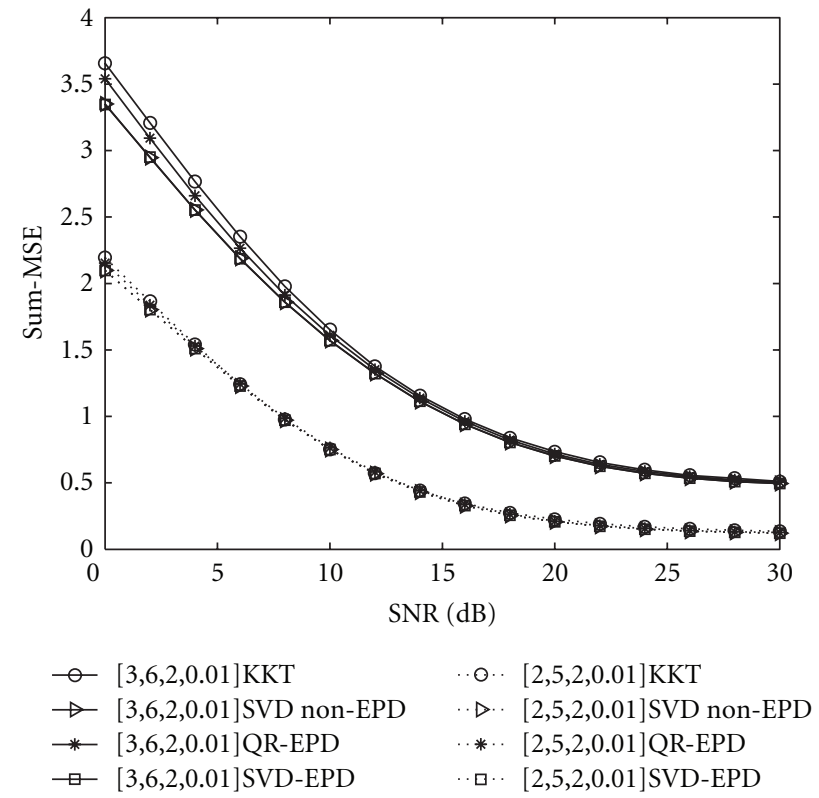

(a)

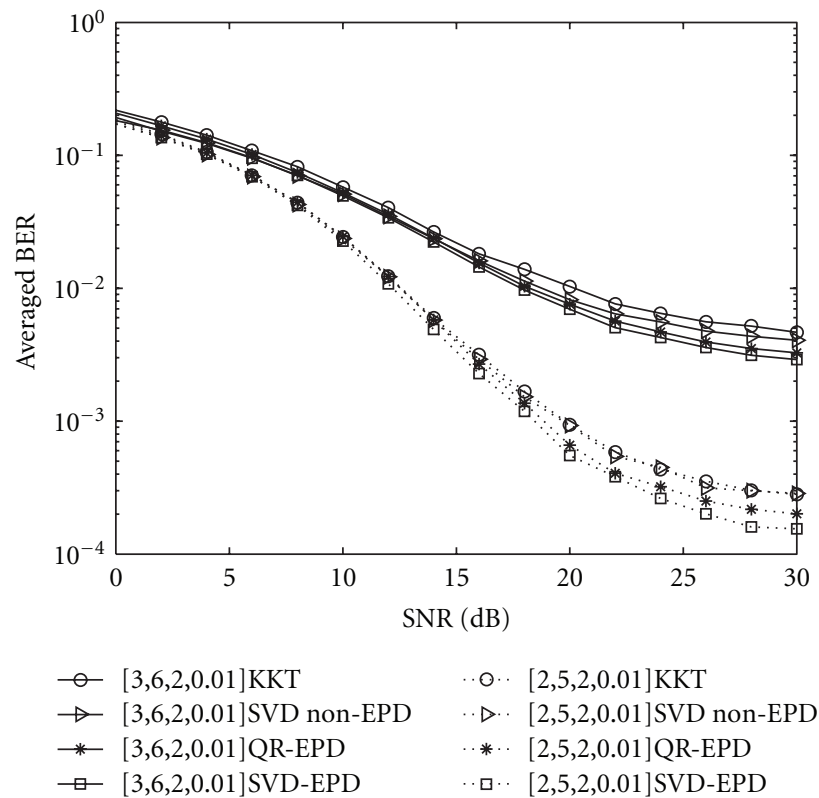

(b)

FIGURE 3: (a) The SMSE and (b) the averaged BER comparisons between the proposed QR-EPD, the SVD-EPD, the SVD non-EPD, and the KKT algorithms for $\sigma_{\mathrm{E}}^{2}=0.01$.

$\left[K, M, N, \sigma_{\mathrm{E}}^{2}\right]$ under the condition of limited CSI, that is, $\sigma_{\mathrm{E}}^{2} \in\{0.01,0.05\}$. It can be observed from figures that the SMSE and the averaged BER performance of all these schemes degrade dramatically as $\sigma_{\mathrm{E}}^{2}$ increases. Additionally, the performance deviation of these schemes between these two cases of $K N<M$ and $K N=M$ is increased as $\sigma_{\mathrm{E}}^{2}$ increases. However, it can be seen from Figures 3 and 4 that the SMSE and the average BER performance of the proposed QR-EPD scheme are still close to the SVD-EPD and superior 


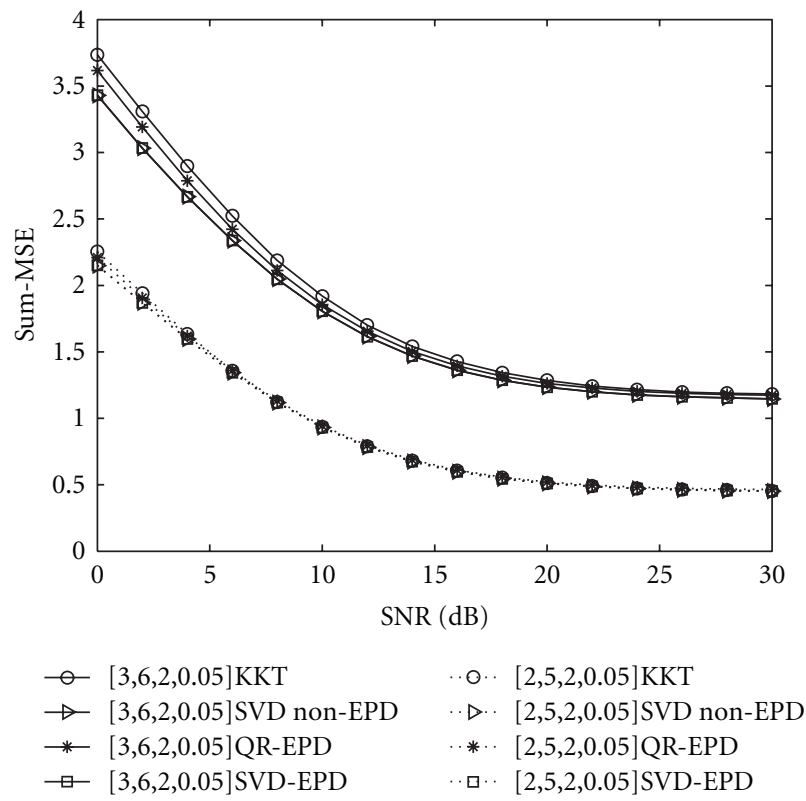

(a)

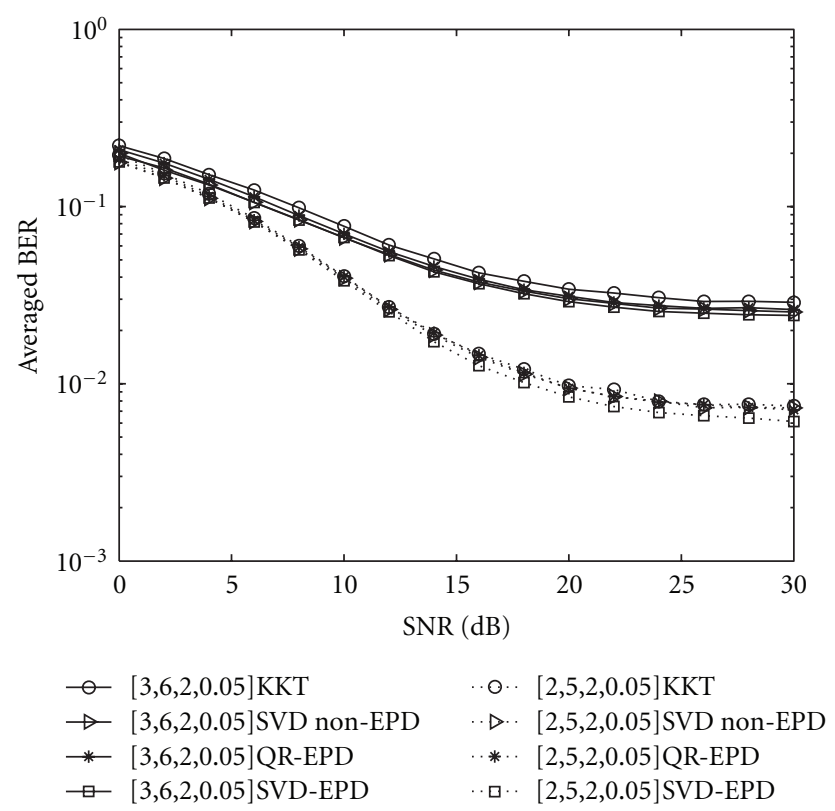

(b)

FIgURE 4: (a) The SMSE and (b) the averaged BER comparisons between the proposed QR-EPD, the SVD-EPD, the SVD non-EPD, and the KKT algorithms for $\sigma_{\mathrm{E}}^{2}=0.05$.

than those of the SVD non-EPD and the KKT techniques when the imperfect CSI is assumed.

\section{Conclusions}

In this paper, a joint linear precoder-decoder pair is considered for uplink multiuser MIMO wireless systems with limited CSI at both the transmitters and the receivers. Instead of counting on complex iterative-based algorithms, the computationally efficient noniterative QR-based linear transceiver pair is proposed. In addition, an EPD scheme is applied to adjust transmit power allocation of each mobile station between its symbols under the sum-power constraint. Simulation results, compared with those of the existing iterative-based transceiver designs, have shown that the proposed QR-EPD scheme is capable of achieving a superior SMSE and enhance the average BER performance simultaneously without compromising the computational complexity.

\section{References}

[1] E. Telatar, "Capacity of multi-antenna Gaussian channels," European Transactions on Telecommunications, vol. 10, no. 6, pp. 585-595, 1999.

[2] S. Serbetli and A. Yener, "Transceiver optimization for multiuser MIMO systems," IEEE Transactions on Signal Processing, vol. 52, no. 1, pp. 214-226, 2004.

[3] J. Zhang, Y. Wu, S. Zhou, and J. Wang, "Joint linear transmitter and receiver design for the downlink of multiuser MIMO systems," IEEE Communications Letters, vol. 9, no. 11, pp. 991993, 2005.

[4] A. Mezghani, R. Hunger, M. Joham, and W. Utschick, "Iterative THP transceiver optimization for multi-user MIMO systems based on weighted sum-MSE minimization," in Proceedings of the 7th IEEE Workshop on Signal Processing Advances in Wireless Communications (SPAWC'06), July 2006.

[5] H. Sampath, P. Stoica, and A. Paulraj, "Generalized linear precoder and decoder design for MIMO channels using the weighted MMSE criterion," IEEE Transactions on Communications, vol. 49, no. 12, pp. 2198-2206, 2001.

[6] A. Scaglione, P. Stoica, S. Barbarossa, G. B. Giannakis, and H. Sampath, "Optimal designs for space-time linear precoders and decoders," IEEE Transactions on Signal Processing, vol. 50, no. 5, pp. 1051-1064, 2002.

[7] J. W. Huang, E. K. S. Au, and V. K. N. Lau, "Linear precoder and equalizer design for uplink multiuser MIMO systems with imperfect channel state information," in Proceedings of the IEEE Wireless Communications and Networking Conference (WCNC '07), pp. 1296-1301, March 2007.

[8] S. J. Leon, Linear Algebra with Applications, Prentice Hall, Upper Saddle River, NJ, USA, 7th edition, 2005.

[9] C. H. Pan, "An efficient QR-based selection criterion for selecting an optimal precoding matrix employed in a simplistic MIMO detection," WSEAS Transactions on Signal Processing, vol. 5, no. 7, pp. 271-282, 2009.

[10] H. Sun, J. H. Manton, and Z. Ding, "Progressive linear precoder optimization for MIMO packet retransmissions," IEEE Journal on Selected Areas in Communications, vol. 24, no. 3, pp. 448-456, 2006.

[11] M. Ding and S. D. Blostein, "Relation between joint optimizations for multiuser MIMO uplink and downlink with imperfect CSI," in Proceedings of the IEEE International Conference on Acoustics, Speech and Signal Processing (ICASSP '08), pp. 3149-3152, April 2008. 

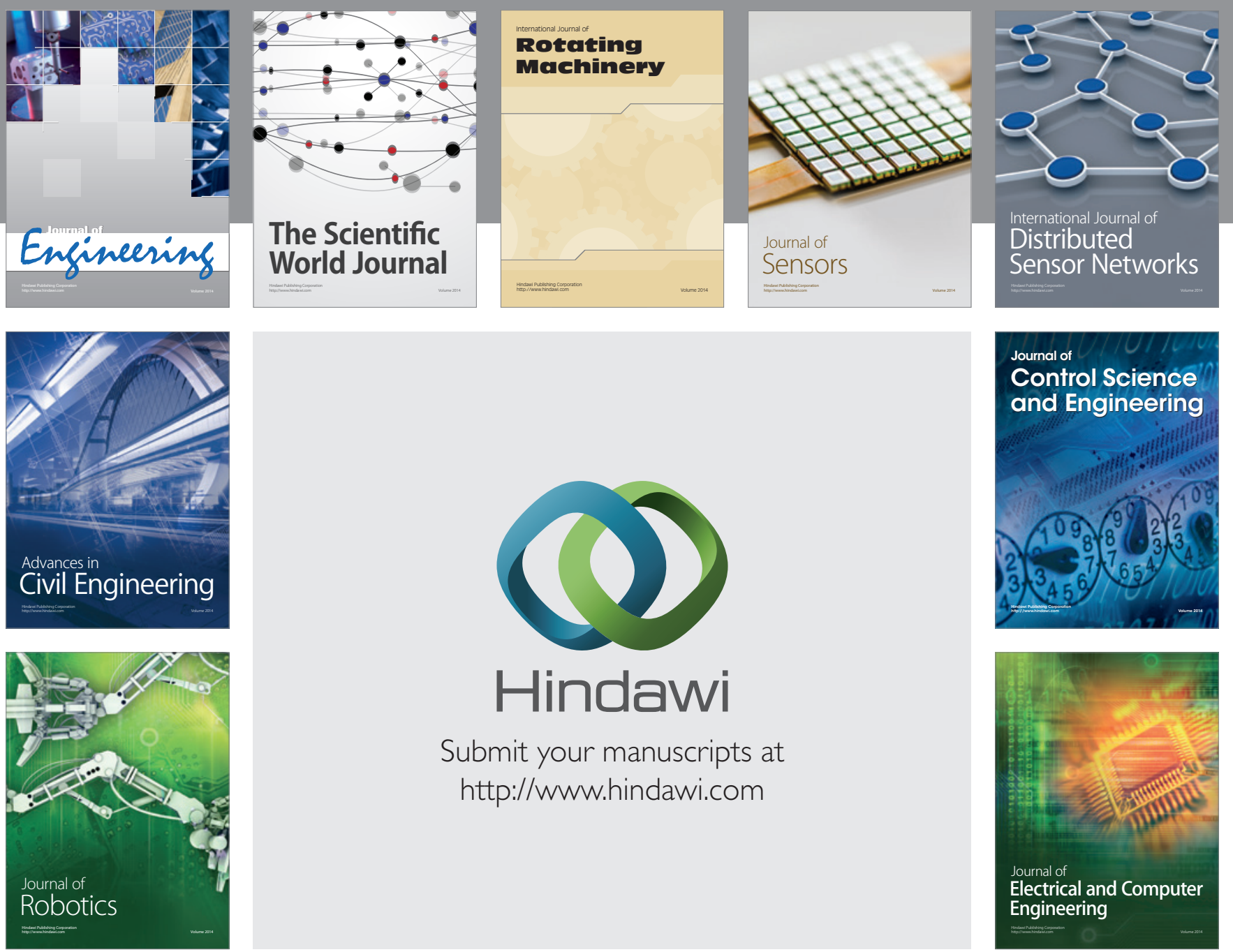

Submit your manuscripts at

http://www.hindawi.com
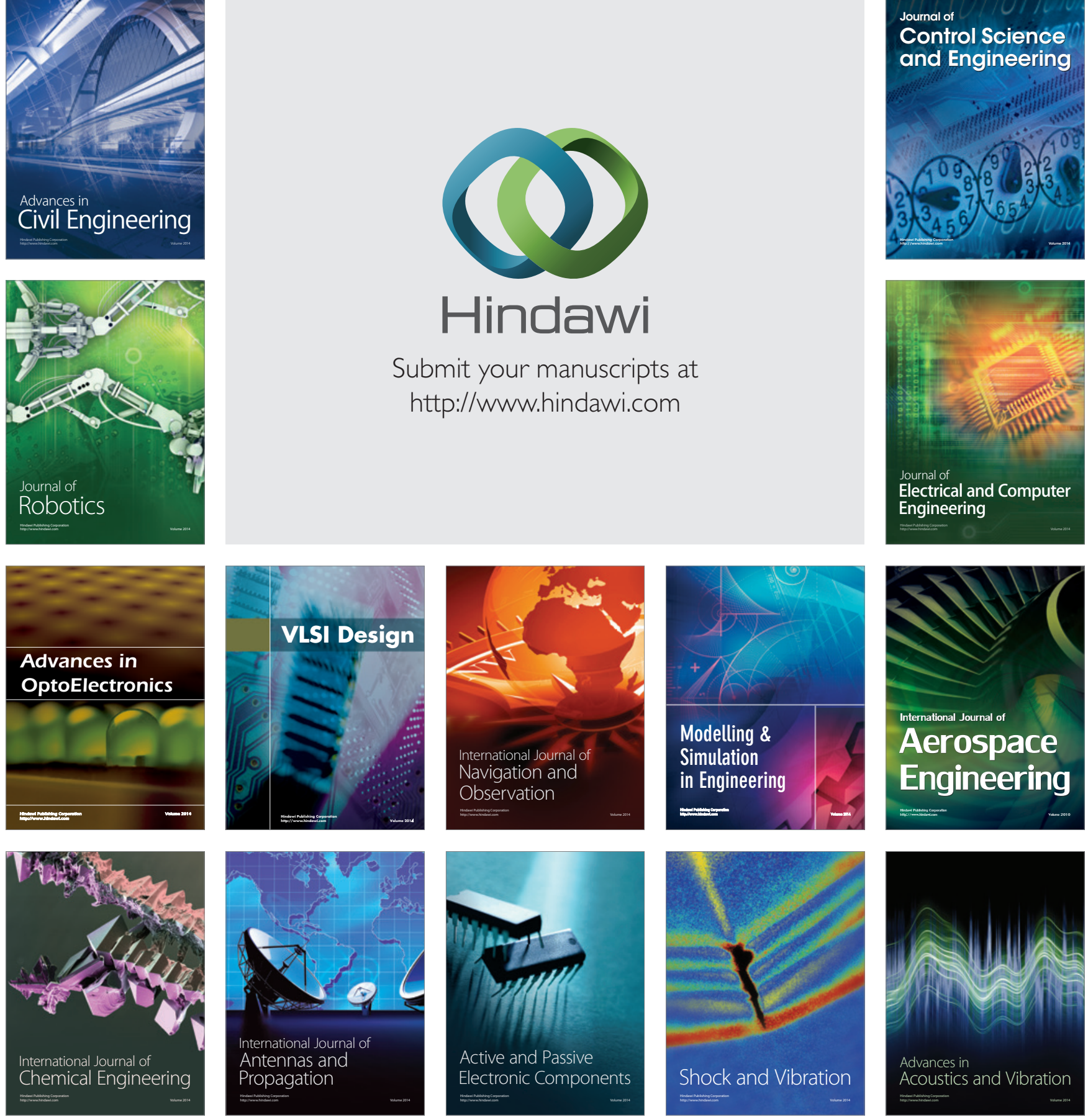